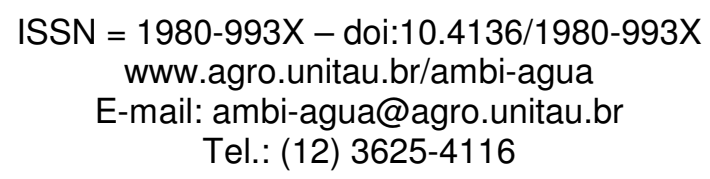

\title{
Caracterização geoelétrica de contaminantes provenientes da decomposição de resíduos sólidos domiciliares \\ (doi:10.4136/ambi-agua.90)
}

\author{
César Augusto Moreira ${ }^{1}$; Antonio Celso de Oliveira Braga ${ }^{2}$ \\ ${ }^{1}$ Universidade Federal do Pampa - UNIPAMPA - Campus Caçapava do Sul (RS) \\ ${ }^{2}$ Universidade Estadual Paulista - UNESP, Campus Rio Claro (SP) \\ Universidade Estadual Paulista - UNESP \\ E-mail: cesargeologia@yahoo.com.br; acobraga@rc.unesp.br
}

\section{RESUMO}

Este trabalho apresenta os resultados da aplicação do método geofísico de Eletrorresistividade, na determinação de área de percolação de material lixiviado proveniente de resíduos sólidos domiciliares, dispostos em aterro do tipo vala. $\mathrm{Na}$ ausência de poços de monitoramento ou outras técnicas diretas de investigação ambiental, estudos geológicos em conjunto com a aplicação de técnicas geofísicas são uma alternativa para caracterização e monitoramento de materiais lixiviados. A ausência de valores de resistividade inferiores a 100 $\Omega . m$ na linha referência a $4 \mathrm{~m}$ de profundidade e a ocorrência desses valores nas linhas realizadas sobre as valas de resíduos e nas adjacências, indica a presença de chorume. Essa relação está associada ao elevado teor de sólidos totais dissolvidos que caracterizam o contaminante em questão, cuja presença no solo resulta em redução nos valores de resistividade elétrica.

Palavras-chave: aterro sanitário; chorume; geofísica; resistividade elétrica.

\section{Geoelectrical characterization of contaminants from domiciliary solids waste decomposition}

\begin{abstract}
This paper presents the results of the Electrical Resistivity geophysical method applied to determine the area of leaching migration originated from solids waste decomposition, disposed in ditch type landfill. When the monitoring of wells or other direct techniques of the environmental investigation is not possible, the geological characterization, supported by geophysics techniques is an alternative for the assessment and monitoring leached materials. The absence of values below $100 \Omega . \mathrm{m}$ in the reference line at $4 \mathrm{~m}$ of depth and the occurrence of these values in the residues ditches and its surroundings indicate the presence of leachate. This relation is a result of the high content of total dissolved solids that characterize the studied contaminant that reduces the electric resistivity.
\end{abstract}

Keywords: landfill; leachate; geophysics; electric resistivity.

\section{INTRODUÇÃO}

A avaliação de contaminações provocadas por aterros municipais de resíduos sólidos envolvem a análise integrada do meio físico. Os aspectos fundamentais necessários para a caracterização de tais áreas sugerem estudos hidrogeológicos, geológicos e pedológicos. 
MOREIRA, C. A.; BRAGA, A. C. O. Caracterização geoelétrica de contaminantes provenientes da decomposição de resíduos sólidos domiciliares. Ambi-Agua, Taubaté, v. 4, n. 2, p. 106-114, 2009. (doi:10.4136/ambi-agua.90)

O acompanhamento e monitoramento das contaminações eventualmente presentes requerem a detecção, caracterização, acompanhamento e prognósticos sobre o fluxo das plumas de contaminação geradas no solo e seus possíveis efeitos aos ecossistemas.

Análises químicas em amostras de solo e água subterrânea, caracterizadas como formas diretas de investigação, são ferramentas tradicionalmente empregadas no estudo de áreas impactadas por contaminações e são necessárias para a proposição e estabelecimento de técnicas de tratamento e remediação. Entretanto, esses tipos de análises apresentam caráter pontual e suas avaliações diante das descontinuidades intrínsecas ao ambiente geológico, muitas vezes dificultam a interpretação.

A aplicação dos métodos geofísicos em estudos ambientais, essencialmente na caracterização e monitoramento de áreas impactadas por contaminantes, é crescente diante da demanda de novas técnicas de investigação ambiental. Esse tipo de análise é caracterizado como método indireto de investigação, pois realiza medidas de parâmetros físicos relacionados a propriedades elétricas, mecânicas, térmicas, magnéticas e/ou gravitacionais, características dos materiais geológicos. Dentre estes, a resistividade elétrica é o parâmetro mais susceptível a alterações em presença de contaminantes, que podem ser perceptíveis ao instrumental geofísico.

A possibilidade de aquisição de dados em larga escala a um custo relativamente baixo por meio de instrumentos de medição permite uma avaliação mais adequada do ambiente, principalmente, quando integrado a resultados diretos de investigação. Por outro lado, na ausência destes, as técnicas geofísicas podem auxiliar no diagnóstico de áreas contaminadas sujeitas a alterações nas propriedades físicas do ambiente impactado.

Este trabalho apresenta os resultados da aplicação do método de Eletrorresistividade, por meio da técnica de caminhamento elétrico, na determinação da área contaminada por chorume proveniente de decomposição de resíduos sólidos domiciliares depositados no aterro municipal de Cordeirópolis, Estado de São Paulo.

\section{REVISÃO BIBLIOGRÁFICA}

\subsection{Método Geofísico}

O trabalho de Elis (1998) descreve as possibilidades de aplicação dos métodos geofísicos elétricos no estudo de áreas de disposição de resíduos e suas limitações na contaminação gerada. Os resultados indicam o método de Eletrorresistividade como a ferramenta mais indicada aos objetivos propostos, principalmente, devido à grande versatilidade, facilidade e rapidez na aquisição de dados em campo. O método de Potencial Espontâneo demonstrou ser bastante adequado ao estudo de alvos específicos, como atividade geoquímica resultante da degradação dos resíduos orgânicos e o fluxo em zonas fraturadas.

Por meio da aplicação conjunta das técnicas de sondagem elétrica vertical, caminhamento elétrico - para leitura dos parâmetros resistividade e cargabilidade, e o método de Potencial Espontâneo em arranjo gradiente, Elis e Zuquette (2001) caracterizam uma área de lançamento de resíduos de curtume ricos em cromo, em Franca (SP). Os resultados indicam que a resistividade elétrica é o parâmetro mais promissor na identificação de zonas anômalas associadas ao fluxo de resíduos, enquanto que a cargabilidade não revela relação de contraste entre áreas impactadas e não impactadas. Os dados de potencial espontâneo auxiliaram na determinação de caminhos preferenciais de fluxo d'água subterrânea, sem indicar atividade geoquímica nas áreas contaminadas.

No aterro sanitário de Bauru (Brasil), Lago et al. (2006) aplicaram os métodos de eletrorresistividade e polarização induzida no estudo das relações espaciais entre a área de disposição de resíduos e o ambiente natural. Os resíduos contidos em valas apresentam baixa 
MOREIRA, C. A.; BRAGA, A. C. O. Caracterização geoelétrica de contaminantes provenientes da decomposição de resíduos sólidos domiciliares. Ambi-Agua, Taubaté, v. 4, n. 2, p. 106-114, 2009. (doi:10.4136/ambi-agua.90)

resistividade e elevados valores de cargabilidade, depositados em cotas no terreno acima do nível freático. Os autores definem intervalos de zona saturada e zona insaturada e apontam áreas contaminadas dentro da zona saturada, caracterizadas por baixos valores de resistividade e de cargabilidade.

Com o objetivo de estimar a extensão e espessura de resíduos e definir a profundidade do nível freático contaminado do aterro sanitário de Mallard North - Ilinóis (Estados Unidos), Carpenter et al. (1990) utilizaram a técnica de sondagem elétrica vertical com arranjos Wenner e Schlumberger. Os resultados indicam o arranjo Schlumberger como o mais adequado ao estudo em áreas contaminadas, pois permite definir estratos de cobertura de resíduos (15 $\Omega . \mathrm{m}$ a $30 \Omega . \mathrm{m})$, estratos com resíduos insaturados (9 $\Omega . \mathrm{m}$ a $19 \Omega . \mathrm{m})$ e o nível saturado de acúmulo de chorume.

A integração de análise estrutural, condutividade hidráulica e resistividade por meio de sondagens elétricas verticais, possibilitaram a Frohlich et al. (1996), uma estimativa das propriedades hidráulicas do maciço fraturado onde está localizado o aterro sanitário Central de Rhode Island (Estados Unidos). A definição de caminhos preferenciais de fluxo d'água no maciço foi possível devido à baixa resistividade elétrica associada a planos de fratura. A correlação linear inversa entre condutividade hidráulica e resistividade elétrica das camadas estudadas, sugere que íons presentes na água contida nas fraturas são condutores de corrente elétrica.

A caracterização do aterro de East Anglia (Reino Unido), em termos de resistividade e cargabilidade por Aristodemou e Thomas-Betts (2000), revela aumento nos valores de cargabilidade na área do aterro dentro da zona saturada e com o aumento da profundidade, ao passo que tais intervalos foram caracterizados por valores de resistividade em torno de 0,3 $\Omega . m$ para a zona saturada e variando entre $0,3 \Omega . \mathrm{m}$ e $50 \Omega . \mathrm{m}$ com o aumento da profundidade. Ambos os parâmetros permitiram distinguir de forma clara as regiões afetadas por contaminantes.

$\mathrm{Na}$ caracterização geoquímica e geofísica do aterro sanitário municipal de Camacchio (Itália), Abu-Zeid at al. (2004) utilizaram os métodos de Eletrorresistividade e Polarização Induzida (IP), na tentativa de esclarecer a resposta IP geralmente observada em aterros e suas áreas adjacentes. As áreas adjacentes ao aterro com elevada permeabilidade apresentam baixa resistividade aparente pela presença de água rica em sais, concomitante a valores elevados de cargabilidade, provavelmente devido a efeito de polarização de membrana. Resultados obtidos sobre os intervalos com resíduos também indicam baixos valores de resistividade e elevados valores de cargabilidade, nesse caso associado à presença de metais sob processos de oxidação/redução, identificados em análises químicas, sob a forma de elementos metálicos em solução e em suspensão na zona saturada.

A combinação dos métodos geofísicos da Eletrorresistividade, Radar de Penetração no Solo (GPR) e Very Low Frequence (VLF) identificam claramente a distribuição de zonas de baixa condutividade elétrica em solo e na rocha subjacente no aterro sanitário Tveta (Suécia), segundo Olofsson et al. (2006). Análises químicas na água subterrânea do aterro indicam elevada quantidade de sais dissolvidos, que confirmam a suposta composição eletrolítica do aquífero a partir dos dados geofísicos.

\subsection{Caracterização geoquímica do Chorume}

Atenuação é um termo frequentemente aplicado a redução das concentrações de oxigênio dissolvido em plumas de contaminação, que incluem processos: físicos - diluição; físico/químicos - sorção, troca iônica; químicos - precipitação; microbial - degradação (Christensen et al., 2001).

Plumas de contaminação provenientes de aterros são frequentemente caracterizadas por quatro grupos de poluentes (Christensen et al., 1994): 
MOREIRA, C. A.; BRAGA, A. C. O. Caracterização geoelétrica de contaminantes provenientes da decomposição de resíduos sólidos domiciliares. Ambi-Agua, Taubaté, v. 4, n. 2, p. 106-114, 2009. (doi:10.4136/ambi-agua.90)

- Matéria orgânica dissolvida, expressa pela demanda química de oxigênio ou carbono orgânico total, incluindo $\mathrm{CH}_{4}$, ácidos graxos voláteis e compostos mais refratários como húmicos e fúlvicos.

- Componentes inorgânicos: $\mathrm{Ca}, \mathrm{Mg}, \mathrm{Na}, \mathrm{K}, \mathrm{NH}_{4}{ }^{+}, \mathrm{Fe}, \mathrm{Mn}, \mathrm{Cl}, \mathrm{SO}_{4}{ }^{2+}, \mathrm{HCO}_{3}{ }^{-}$.

- Metais pesados: $\mathrm{Cd}, \mathrm{Cr}, \mathrm{Cu}, \mathrm{Pb}, \mathrm{Ni}$ e $\mathrm{Zn}$.

- Compostos orgânicos derivados de petróleo, que incluem hidrocarbonetos aromáticos, fenóis e compostos clorados alifáticos.

Outros compostos podem ser encontrados nesse ambiente, como B, As, $\mathrm{Se}, \mathrm{Ba}, \mathrm{Li}, \mathrm{Hg}$, e Co, sempre em concentrações muito baixas e de importância secundária. A detecção e as concentrações desses elementos ou compostos podem variar devido a mudanças das fases de oxidação/redução e/ou alterações sazonais.

Processos de oxidação-redução exercem grande influência na geoquímica de elementos orgânicos e inorgânicos em aquíferos, com controle da estruturação química. Em geral, o potencial redox aumenta da parte central para as extremidades da área contaminada.

A condutividade elétrica também pode ser medida em termos de sólidos totais dissolvidos, devido à associação do íon cloreto como indicador conservativo da presença de chorume em amostras de água (Baedecker e Apgar, 1984). O trabalho de Bell e Jermy (1995), dentre muitos outros, descreve a relação direta entre queda no teor de sólidos totais dissolvidos e redução da resistividade elétrica com o distanciamento do aterro.

\section{MATERIAL E MÉTODOS}

\subsection{Localização e características da área de estudos}

Os estudos foram realizados no aterro municipal de Cordeirópolis, localizado na Rodovia Cássio de Freitas Levi, km 4.5, Estado de São Paulo, Brasil (Figura 1). O município de Cordeirópolis possui cerca de 17.000 habitantes, cuja economia está baseada na produção de pisos cerâmicos e cultivo de cana-de-açúcar. A produção de lixo doméstico é de $6 \mathrm{~m}^{3} /$ dia.

A área de estudos é um aterro controlado do tipo vala, para deposição apenas de resíduos sólidos domiciliares. O sistema de operação consiste em valas com $5 \mathrm{~m}$ de profundidade, $4 \mathrm{~m}$ de largura e $80 \mathrm{~m}$ de comprimento, para deposição de resíduos diretamente sobre o solo e subsequente cobertura com uma camada de $1 \mathrm{~m}$ de solo. Apresenta $48.400 \mathrm{~m}^{2}$ de área disponível, com início das atividades em novembro de 2001 e vida útil prevista para 20 anos.

A topografia da área é bastante plana, com declive médio de $0,5 \%$ sentido sudeste e altitude entre $660 \mathrm{~m}$ e $659 \mathrm{~m}$. O aterro é cercado por fazendas de cultivo de cana-de-açúcar.

O substrato é constituído por solo argiloso com $10 \mathrm{~m}$ de espessura, sotoposto a um corpo de diabásio com $15 \mathrm{~m}$ de espessura. Abaixo dele ocorrem arenitos finos e siltitos pertencentes à Formação Tatuí. Ensaios de peneiramento e análise granulométrica conjunta de minerais magnéticos revelam a concentração de $60 \%$ de minerais metálicos no solo, essencialmente óxidos de ferro sob a forma de lâminas lateríticas milimétricas, glóbulos de goetita e cristais parcialmente alterados de magnetita.

O nível freático está localizado a uma profundidade média de $50 \mathrm{~m}$, com sentido de fluxo para sudeste, determinados por meio de sondagens elétricas verticais. Ensaios de condutividade hidráulica saturada de campo apontam valores entre $10^{-5} \mathrm{~cm} / \mathrm{s} \mathrm{a} 10^{-3} \mathrm{~cm} / \mathrm{s}$ para o solo da área. Não existem poços instalados na área ou quaisquer outros sistemas de monitoramento ambiental do solo ou água subterrânea. 
MOREIRA, C. A.; BRAGA, A. C. O. Caracterização geoelétrica de contaminantes provenientes da decomposição de resíduos sólidos domiciliares. Ambi-Agua, Taubaté, v. 4, n. 2, p. 106-114, 2009. (doi:10.4136/ambi-agua.90)

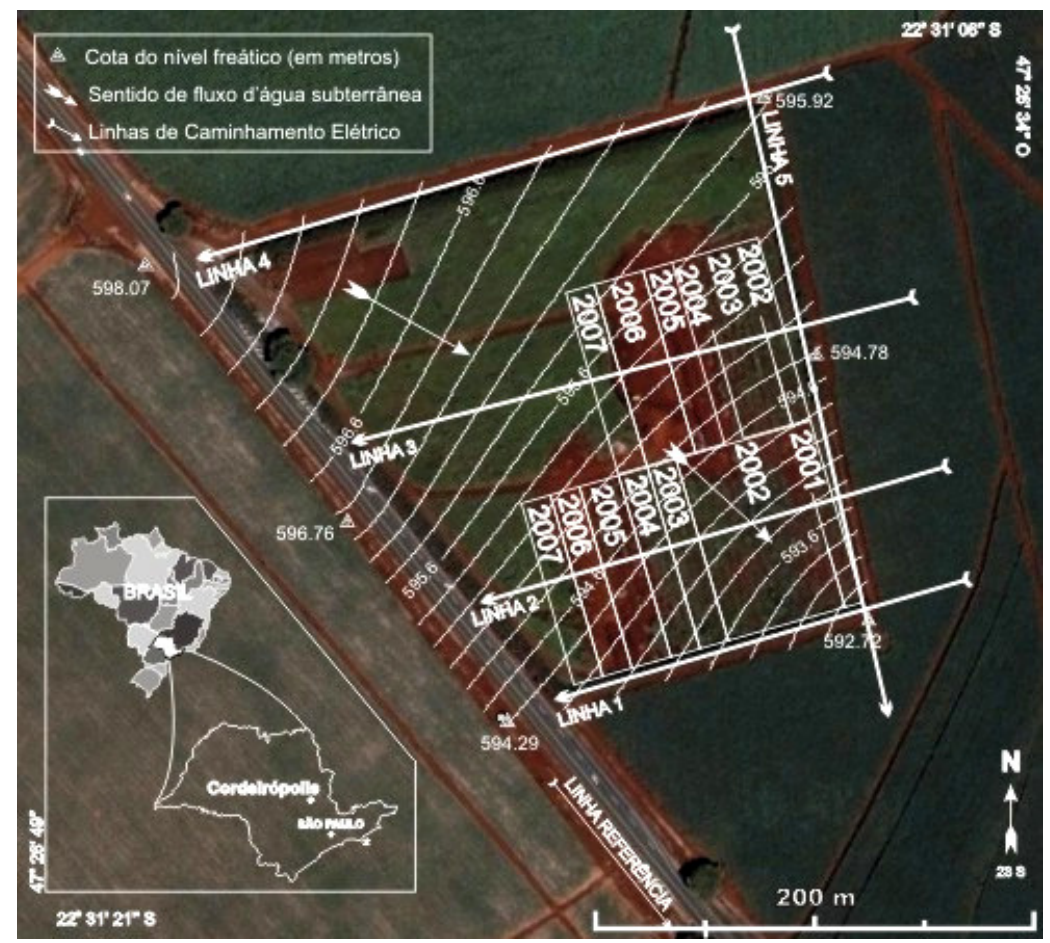

Figura 1. Localização da área de estudo, com posição das linhas de caminhamento elétrico, período de fechamento das valas de resíduos, potenciometria e sentido de fluxo d'água subterrânea.

\subsection{Desenvolvimento dos trabalhos de campo}

A aquisição de dados geofísicos utilizou a técnica de caminhamento elétrico, por meio de do arranjo dipolo-dipolo. Esse arranjo consiste na instalação de pares de eletrodos metálicos ao longo da linha de investigação, para injeção de corrente elétrica no solo por meio de eletrodos de corrente e posterior leitura do potencial gerado, por meio de eletrodos de potencial (Orellana, 1972). A disposição adequada dos pares de eletrodos de corrente e potencial na superfície do terreno permitem leituras de resistividade elétrica para diversos níveis de profundidade na linha investigada. Este trabalho utilizou o arranjo dipolo-dipolo com $5 \mathrm{~m}$ de espaçamento entre eletrodos e leituras de resistividade em 8 níveis de profundidade.

$\mathrm{Na}$ área estudada foram realizadas 6 linhas de caminhamento elétrico (Figura 1). As linhas 2 e 3 cruzam as valas de resíduos enterrados, enquanto que as linhas 1, 4 e 5 estão locadas nos limites da área. A linha referência foi disposta em uma área a montante do fluxo d'água subterrânea e separada da área do aterro pelo talude de implantação da rodovia que cruza a área. Esta linha foi realizada para determinação dos valores naturais de resistividade em diversos níveis no terreno, para sua posterior comparação com as linhas que eventualmente sofrem influência de contaminantes provenientes do aterro.

O equipamento utilizado foi o resistivímetro Terrameter SAS 4000, fabricado pela ABEM Instruments (Suécia), com resolução de $1 \mu \mathrm{V}$. Esse equipamento é calibrado para medidas de resistividade por meio de ciclos periódicos de corrente elétrica alternada e de baixa frequência, procedimento que permite a filtragem de ruídos do sinal adquirido. A razão V/I, ou seja, potencial medido (V) pela corrente (I) transmitida permite leituras diretas de resistência elétrica em ohm $(\Omega)$. A resistência medida, associada ao fator $\mathrm{K}$, associado à geometria dos eletrodos em superfície, resulta no parâmetro resistividade elétrica (Equação 1):

$$
\rho=K \frac{\Delta \mathrm{V}}{\mathrm{I}} \quad \text { Ohm.m }
$$


MOREIRA, C. A.; BRAGA, A. C. O. Caracterização geoelétrica de contaminantes provenientes da decomposição de resíduos sólidos domiciliares. Ambi-Agua, Taubaté, v. 4, n. 2, p. 106-114, 2009. (doi:10.4136/ambi-agua.90)

(*) Os dados foram processados no programa RES2DINV, no qual foram gerados modelos por inversão, resultando em seções geoelétricas 2D (distância versus profundidade) (Loke e Barker, 1996). Durante o processamento, foi realizada a calibração de profundidade em todas as seções modeladas, a partir de informações conhecidas sobre a profundidade de escavação das valas.

Posteriormente, todas as medidas referentes a $4 \mathrm{~m}$ de profundidade foram tabeladas, georreferenciadas e interpoladas no programa Surfer 8.0, a por meio do método de vizinhança.

Nesse método, os grupos iniciais são determinados pelos mais altos coeficientes de associação mútua, ou seja, para a admissão de novos membros é suficiente determinar quais os representantes de maior coeficiente de associação com um dos elementos de determinado grupo (Landim, 2004). Dessa forma, a escolha desse método de interpolação foi baseada na fidelidade aos valores interpolados e limitação da superfície aos valores extremos de entrada.

\section{RESULTADOS E DISCUSSÃO}

O perfil de solo da área é caracterizado pelo aumento crescente de resistividade elétrica com a profundidade, como pode ser observado na seção geoelétrica 2D modelada (Figura 2). Os horizontes até $2 \mathrm{~m}$ de profundidade, onde predomina solo areno-argiloso, apresentam valores de resistividade entre $30 \Omega . \mathrm{m}$ e $300 \Omega . \mathrm{m}$. Entre $2 \mathrm{~m}$ e $5 \mathrm{~m}$ de profundidade, onde predomina solo silto-argiloso, ocorrem valores de resistividade que variam de $300 \Omega$.m e 3000 $\Omega . m$.

A partir de $5 \mathrm{~m}$ ocorre solo saprolítico com fragmentos centimétricos bastante alterados de rocha, envoltos em matriz argilosa. A resistividade desse intervalo varia de $3000 \Omega . \mathrm{m}$ a $8000 \Omega$.m. Abaixo de $10 \mathrm{~m}$ de profundidade ocorre diabásio inalterado, com juntas verticais totalmente ausentes de água.

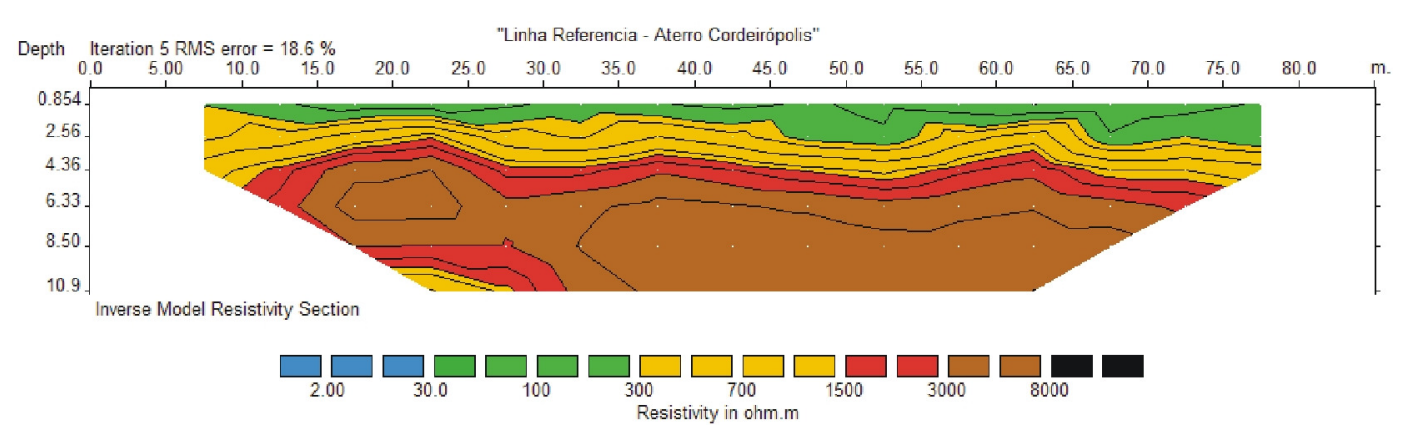

Figura 2. Seção geoelétrica 2D correspondente à linha de referência.

O mapa de integração das linhas processadas (Figura 3) apresenta intervalos de resistividade bastante inferiores aos valores característicos para a área, conforme demonstra a seção de referência (Figura 2). É possível que áreas com resistividade acima de $700 \Omega$.m estejam próximas das condições naturais da área, caracterizada por valores entre $1000 \Omega . m$ a $1500 \Omega$.m para $4 \mathrm{~m}$ de profundidade.

A isolinha central com valores abaixo de $100 \Omega$.m, possivelmente, representa a área com maior concentração de contaminantes, constituído por elevada carga de sólidos totais dissolvidos que caracterizam o liquido lixiviado proveniente da decomposição de resíduos orgânicos.

O formato dessa área anômala sugere que o fluxo de chorume esteja condicionado à direção longitudinal das valas. É possível que o fluxo de chorume dentro das valas seja 
MOREIRA, C. A.; BRAGA, A. C. O. Caracterização geoelétrica de contaminantes provenientes da decomposição de resíduos sólidos domiciliares. Ambi-Agua, Taubaté, v. 4, n. 2, p. 106-114, 2009. (doi:10.4136/ambi-agua.90)

facilitado devido à elevada porosidade e permeabilidade desse ambiente em relação ao constituído pelo solo da área.

A ocorrência de medidas abaixo de $100 \Omega$.m em diversas valas, aparentemente, indica haver uma relação entre tempo de decomposição de resíduos orgânicos e variação da resistividade elétrica, pois esse intervalo de resistividade ocorre nas valas com fechamento entre 2007 e 2005, cruzadas pela linha 3.

Essas valas estão a montante das valas de 2004, 2003 e 2002 cruzadas pela linha 2, nas quais também ocorrem medidas abaixo de $100 \Omega$.m (Figura 3).

Grande parte da linha 1 apresenta medidas abaixo de $300 \Omega$.m, indicando um possível avanço de chorume para além dos limites do aterro. Essa tendência também ocorre na linha 4 , embora com pequena faixa de ocorrência de medidas abaixo de $400 \Omega$.m.

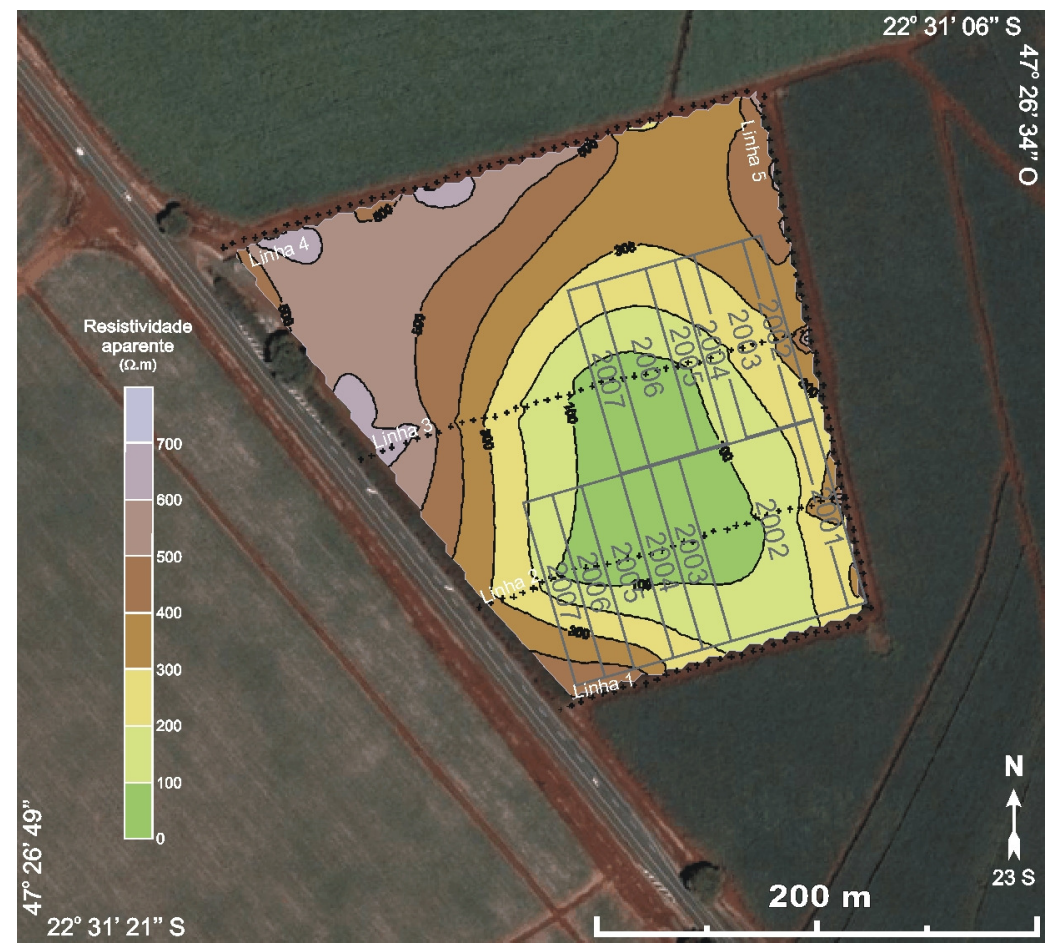

Figura 3. Mapa de resistividade elétrica para $4 \mathrm{~m}$ de profundidade.

\section{CONCLUSÕES}

$\mathrm{Na}$ ausência de poços de monitoramento ou outras técnicas diretas de investigação na área do aterro, a caracterização geológica em conjunto com a aplicação de técnicas geofísica podem ser uma alternativa para detecção e monitoramento de contaminantes.

Entretanto, a determinação dos teores de contaminação requer análises químicas em amostras de solo e água subterrâneas. Portanto, é recomendado que técnicas indiretas de investigação sejam aplicadas de forma complementar em estudos ambientais, seja para caracterização de áreas contaminadas, determinação de áreas de anomalias quanto ao parâmetro físico estudado ou no monitoramento de áreas comprovadamente contaminadas.

A caracterização da área de baixa resistividade foi possível somente após comparação com os padrões de resistividade naturais ao ambiente geológico, definidos pela linha referência. Este é um procedimento necessário em qualquer estudo ambiental para determinação de áreas anômalas, pois os parâmetros físicos medidos pelo instrumental geofísico, especialmente pelo método de Eletrorresistividade, variam com o tipo de 
MOREIRA, C. A.; BRAGA, A. C. O. Caracterização geoelétrica de contaminantes provenientes da decomposição de resíduos sólidos domiciliares. Ambi-Agua, Taubaté, v. 4, n. 2, p. 106-114, 2009. (doi:10.4136/ambi-agua.90)

solo/rocha, características físico-químicas do fluído intersticial, grau de saturação, dentre outros fatores.

Elevadas quantidades de sólidos totais dissolvidos associadas ao contaminante proveniente da decomposição de material orgânico e seu comportamento no ambiente geológico, caracterizado como condutor elétrico, permitiram sua detecção pela aplicação do método de geofísico de Eletrorresistividade.

A relação direta entre baixa resistividade elétrica e elevado teor de sólidos totais dissolvidos, correlacionados aos padrões naturais da área, permitiu a definição de zonas de concentração de chorume por meio do método de Eletrorresistividade.

Ocorrências de medidas de baixa resistividade na linha 1 indicam a percolação de chorume para além dos limites do aterro. Em contrapartida, a definição dessa zona anômala e sua associação com o vetor de fluxo principal, podem subsidiar projetos de remediação ou contenção da área impactada, como a instalação de barreiras biológicas.

\section{AGRADECIMENTOS}

Este trabalho foi realizado com apoio da CAPES - Coordenação de Aperfeiçoamento de Pessoal de Nível Superior e do CNPq - Conselho Nacional de Desenvolvimento Científico e Tecnológico. Agradecemos ao técnico Francisco Manoel Garcia Barrera e ao Sr. Carlos Aparecido Moreira pelo auxílio na aquisição de dados.

\section{REFERÊNCIAS}

ABU-ZEID, N.; BIANCHINI, G.; SANTARATO, G.; VACCARO, C. Geochemical characterization and geophysical mapping of Landfill leachates: the Marozzo canal case study (NE Italy). Environmental Geology, n 45, p. 439-447, 2004.

ARISTODEMOU, E.; THOMAS-BETTS, A. DC resistivity and induced polarization investigations at waste disposal sites and its environments. Journal of Applied Geophysics, v. 44, p. 275-302, 2000.

BAEDECKER, M. J.; APGAR, M. A. Hydrogeochemical studies at a landfill in Delaware. Washington: Groundwater Contamination, National Academic Press, 1984. p. 127-138.

BELL, F. G.; JERMY, C. A. A seepage problem associated with an old landfill in the greater Durban area. In: SARSBY, R. W. (Ed.). Waste Disposal by Landfill: GREEN'93. Rotterdam: A. A. Balkema, 1995. p. 607-615.

CARPENTER, P. J.; KAUFMANN, R. S.; PRICE, B. Use of Resistivity Soundings to Determine Landfill Structure. Ground Water, v. 28, p. 569-575, 1990.

CHRISTENSEN, T. H.; KJELDSEN, P.; ALBRECHTSEN, H. J.; HERON, G.; NIELSEN, P. H.; BJERG, P. L.; HOLM, P. E. Attenuation of landfill leachate pollutants in aquifers. Critical Review in Environmental Science and Technology, n 24, p. 119-202, 1994.

CHRISTENSEN, T. H.; KJELDSEN, P.; BJERG, P. L.; JENSEN, D. L.; CHRISTENSEN, J. B.; BAUN, A.; ALBRECHTSEN, H. J.; HERON, G. Biogeochemistry of landfill leachate plumes. Applied Geochemistry, n 16, p. 659-718, 2001.

ELIS, V. R. Avaliação da aplicabilidade de métodos elétricos de prospecção geofísica no estudo de áreas utilizadas para disposição de resíduos. 1998. 264f. Tese (Doutorado em Recursos Minerais e Hidrogeologia) - Instituto de Geociências e Ciências Exatas, Universidade Estadual Paulista, Rio Claro, 1998. 
MOREIRA, C. A.; BRAGA, A. C. O. Caracterização geoelétrica de contaminantes provenientes da decomposição de resíduos sólidos domiciliares. Ambi-Agua, Taubaté, v. 4, n. 2, p. 106-114, 2009. (doi:10.4136/ambi-agua.90)

ELIS V. R.; ZUQUETTE, L. V. Aplicação integrada de métodos geoeléctricos em áreas de disposição de resíduos industriais: cidade de Franca, Brasil. Geotecnia, n 92, p. 47-69, 2001.

FROHLICH, R. K.; FISHER, J. J.; SUMMERLY, E. Electric-hydraulic conductivity correlation in fractured crystalline bedrock: Central Landfill, Rodhe Island, USA. Journal of Applied Geophysics, n. 35, p. 249-259, 1996.

LAGO, A. L.; ELIS, V. R.; GIACHETI, H. L. Aplicação integrada de métodos geofísicos em uma área de disposição de resíduos sólidos urbanos em Bauru-SP. Revista Brasileira de Geofísica, n24, v. 3, p. 357-374, 2006.

LANDIM, P. M. B. Análise estatística de dados geológicos. 2. ed. São Paulo: Edunesp, , 2004. 253p.

LOKE, M. H.; BARKER, R. D. Rapid least-squares inversion of apparent resistivity pseudosections by a quasi-Newton method. Geophysical Prospecting, n. 44, p. 131$152,1996$.

OLOFSSON, B.; JERNBERG, H.; ROSENQVIST, A. Tracing leachates at waste sites using geophysical and geochemical modeling. Environmental Geology, n 49, p. 720-732, 2006.

ORELLANA, E. Prospeccion geoelectrica en corriente continua. Madrid: Paraninfo, 1972. 523 p. (Biblioteca Técnica Philips). 